\title{
Polarimetric Calibration and Quality Assessment of the GF-3 Satellite Images
}

\author{
Yonglei Chang ${ }^{1}$, Pingxiang $\mathrm{Li}^{1}{ }^{1, *}$, Jie Yang ${ }^{1}$, Jinqi Zhao ${ }^{1}$ (D), Lingli Zhao ${ }^{2}$ and Lei Shi ${ }^{1}$ (D) \\ 1 State Key Laboratory of Information Engineering in Surveying, Mapping and Remote Sensing, \\ Wuhan University, Wuhan 430079, China; changyonglei@whu.edu.cn (Y.C.); yangj@whu.edu.cn (J.Y.); \\ masurq@whu.edu.cn (J.Z.); shi.lei@whu.edu.cn (L.S.) \\ 2 School of Remote Sensing and Engineering, Wuhan University, Wuhan 430079, China; \\ zhaolingli@whu.edu.cn \\ * Correspondence: pxli@whu.edu.cn; Tel.: +86-138-0713-4352
}

Received: 31 December 2017; Accepted: 26 January 2018; Published: 30 January 2018

\begin{abstract}
The GaoFen-3 (GF-3) satellite is the first fully polarimetric synthetic aperture radar (SAR) satellite designed for civil use in China. The satellite operates in the C-band and has 12 imaging modes for various applications. Three fully polarimetric SAR (PolSAR) imaging modes are provided with a resolution of up to $8 \mathrm{~m}$. Although polarimetric calibration (PolCAL) of the SAR system is periodically undertaken, there is still some residual distortion in the images. In order to assess the polarimetric accuracy of this satellite and improve the image quality, we analyzed the polarimetric distortion errors and performed a PolCAL experiment based on scattering properties and corner reflectors. The experiment indicates that the GF-3 images can meet the satellite's polarimetric accuracy requirements, i.e., a channel imbalance of $0.5 \mathrm{~dB}$ in amplitude and \pm 10 degrees in phase and a crosstalk accuracy of $-35 \mathrm{~dB}$. However, some images still contain residual polarimetric distortion. The experiment also shows that the residual errors of the GF-3 standard images can be diminished after further PolCAL, with a channel imbalance of $0.26 \mathrm{~dB}$ in amplitude and \pm 0.2 degrees in phase and a crosstalk accuracy of $-42 \mathrm{~dB}$.
\end{abstract}

Keywords: polarimetric calibration; PolSAR; GaoFen-3; image quality; reflection symmetry

\section{Introduction}

The GaoFen-3 (GF-3) satellite, which operates in the C-band, is the first fully polarimetric synthetic aperture radar (PolSAR) imaging satellite in China [1]. It was launched on 10 August 2016 and has a design life of eight years. The satellite has 12 imaging modes, the most of any SAR satellite in the world. The imaging modes include StripMap mode, ScanSAR mode, Spotlight mode, wave imaging mode, and ultra-fine strip mode [2]. The details of the different imaging modes are provided in Table 1. GF-3 can achieve an image width of $650 \mathrm{~km}$ at a resolution of $500 \mathrm{~m}$ and a 10-km image width at a resolution of $1 \mathrm{~m} \mathrm{[3].} \mathrm{Therefore,} \mathrm{the} \mathrm{satellite} \mathrm{can} \mathrm{serve} \mathrm{many} \mathrm{industries} \mathrm{and} \mathrm{research} \mathrm{applications,}$ such as disaster monitoring, water conservancy, meteorological observation, marine monitoring, and global environmental studies. Furthermore, the system also has three fully polarimetric SAR imaging modes, making it efficient in geographical interpretation and quantitative research [4].

A PolSAR system measures the scattering information of the Earth's surface with different polarimetric microwaves. Generally, it transmits vertical polarization wave [5] and receives echoes in vertical and horizontal polarizations simultaneously. This is followed by transmitting a horizontal polarization wave and, again, receiving echoes in vertical and horizontal polarizations simultaneously. In this way, the system obtains four images of different polarization channel in one area. Generally speaking, PolSAR technology discriminates ground objects by the coherence and difference of these channel images. Therefore, before PolSAR images can be used for further research or applications, 
polarimetric calibration (PolCAL) is needed to ensure the relative amplitude and phase of the different image channels $[5,6]$. The polarization accuracy is very important for PolSAR satellites such as Radarsat-2, Alos-2, TerraSAR-X, and COSMO-SkyMed. The crosstalk accuracy of Radarsat-2 is $-30 \mathrm{~dB}$ and the channel imbalance is $0.5 \mathrm{~dB}$ in amplitude and 5 degrees in phase [7]. The accuracy requirement of Alos-2 is $-30 \mathrm{~dB}$ in crosstalk and $0.4 \mathrm{~dB}$ in channel imbalance amplitude and 5 degrees in channel imbalance phase, and the practical accuracy in some test sites is much better [8]. After the launch of the GF-3 satellite, a series of ground calibration experiments were carried out in Etuoke Banner, Inner Mongolia. Furthermore, the data processing system was improved in March 2017. It is therefore necessary to again assess the polarimetric accuracy of the satellite images.

Table 1. Imaging modes and parameters of the GF-3 satellite.

\begin{tabular}{cccccc}
\hline Imaging Mode & $\begin{array}{c}\text { Incidence Angle } \\
(\mathbf{0})\end{array}$ & $\begin{array}{c}\text { Look } \\
\text { Number }\end{array}$ & $\begin{array}{c}\text { Resolution } \\
(\mathbf{m})\end{array}$ & $\begin{array}{c}\text { Imaging } \\
\text { Width (km) }\end{array}$ & Polarization Mode \\
\hline Spotlight & $20 \sim 50$ & $1 \times 1$ & 1 & 10 & Selective single-polarization \\
Ultra-fine strip & $20 \sim 50$ & $1 \times 1$ & 3 & 30 & Selective single-polarization \\
Fine strip I & $19 \sim 50$ & $1 \times 1$ & 5 & 50 & Selective dual-polarization \\
Fine strip II & $19 \sim 50$ & $1 \times 2$ & 10 & 100 & Selective dual-polarization \\
Standard strip & $17 \sim 50$ & $3 \times 2$ & 25 & 130 & Selective dual-polarization \\
Narrow scan & $17 \sim 50$ & $1 \times 6$ & 50 & 300 & Selective dual-polarization \\
Wide scan & $17 \sim 50$ & $1 \times 8$ & 100 & 500 & Selective dual-polarization \\
global & $17 \sim 53$ & $2 \times(2 \sim 4)$ & 500 & 650 & Selective dual-polarization \\
Full-polarimetric strip I & $20 \sim 41$ & $1 \times 1$ & 8 & 30 & full-polarization \\
Full-polarimetric strip II & $20 \sim 38$ & $3 \times 2$ & 25 & 40 & full-polarization \\
Wave imaging & $20 \sim 41$ & $1 \times 2$ & 10 & 5 & full-polarization \\
\hline \multirow{2}{*}{ Extend $\quad$ Incidence angle I } & $10 \sim 20$ & $3 \times 2$ & 25 & 130 & Selective dual-polarization \\
$\quad$ Incidence angle II & $50 \sim 60$ & $3 \times 2$ & 25 & 80 & Selective dual-polarization \\
\hline
\end{tabular}

The term "PolCAL" in SAR has two meanings. The first is to determine the polarimetric distortion matrices (PDM) of a PolSAR system, which express the polarimetric transformation between transmission and reception. The other is to convert uncalibrated PolSAR data to calibrated data using these PDMs [9]. A lot of research has been done on PolCAL since the first PolSAR system, i.e., Airborne Synthetic Aperture Radar (AIRSAR), was developed [10]. The early PolCAL methods were designed using different kinds of corner reflectors (CRs) or polarimetric active radar calibrators (PARCs) [11]. Subsequently, some particular scattering targets such as bare soil, desert, and grassland have been investigated and taken as PolCAL references [12-14]. Rainforest has also been studied for PolCAL in some research $[9,15]$. The current PolCAL schemes for PolSAR systems usually use both distributed targets and CRs. Although periodical PolCAL has been performed on the GF-3 system, there is still some channel distortion in the distributed images. In order to assess the polarimetric accuracy of the GF-3 satellite and improve the image quality, we analyzed the polarimetric errors and performed PolCAL based on scattering symmetry and ground CRs.

In this paper, the polarimetric errors of the GF-3 system are described in Section 2. Section 3 describes the PolCAL method and the accuracy verification method used in this research. The experiments and results are discussed in Section 4, and the conclusions are presented in Section 5.

\section{Polarimetric Error Analysis for the GF-3 Satellite}

Generally speaking, the data observed by a PolSAR system is in the format of scattering matrix, which is a $2 \times 2$ complex matrix that characterizes the response from one target. However, during the observation, the real scattering matrix is affected by polarimetric distortion. The distortion is made up of the crosstalk of the different channels, the amplitude and phase imbalance between the co-polarization (co-pol) channels (co-imba), the imbalance between the two cross-polarization (cross-pol) channels (cross-imba), the system noise, and the Faraday rotation. The relationship between 
the scattering matrix and the polarimetric distortion can be represented by the PolSAR distortion model [16]:

$$
\begin{aligned}
& \left(\begin{array}{cc}
O_{h h} & O_{h v} \\
O_{v h} & O_{v v}
\end{array}\right)=A\left[\begin{array}{cc}
1 & w \\
u & 1
\end{array}\right]\left[\begin{array}{ll}
k & 0 \\
0 & 1
\end{array}\right]\left[\begin{array}{cc}
\cos \Omega & \sin \Omega \\
-\sin \Omega & \cos \Omega
\end{array}\right]\left(\begin{array}{cc}
S_{h h} & S_{h v} \\
S_{v h} & S_{v v}
\end{array}\right) * \\
& {\left[\begin{array}{rr}
\cos \Omega & \sin \Omega \\
-\sin \Omega & \cos \Omega
\end{array}\right]\left[\begin{array}{ll}
k & 0 \\
0 & 1
\end{array}\right]\left[\begin{array}{ll}
\alpha & 0 \\
0 & 1
\end{array}\right]\left[\begin{array}{ll}
1 & z \\
v & 1
\end{array}\right]+\left[\begin{array}{ll}
N_{h h} & N_{h v} \\
N_{v h} & N_{v v}
\end{array}\right]}
\end{aligned}
$$

In the model, $S_{h h}, S_{h v}, S_{v h}, S_{v v}$ compose the true scattering matrix of the ground object, with each of them representing a polarization channel. $\mathrm{O}_{h h}, \mathrm{O}_{h v}, \mathrm{O}_{v h}, \mathrm{O}_{v v}$ compose the observation matrix gained by the PolSAR system. The $u, w, v, z$ parameters are the crosstalk distortions. The $k$ term is the co-pol channel imbalance error, and $\alpha$ is the cross-pol channel imbalance error. Argument $A$ is the absolute calibration factor that will be used in the radiometric calibration. Parameter $\Omega$ represents the Faraday rotation angle. $N_{h h}, N_{h v}, N_{v h}, N_{v v}$ represent the system noise of the different channels.

\subsection{Crosstalk Error}

Currently, spaceborne PolSAR systems use transmit-receive modules (TRMs) to emit or detect the microwave signals. The system alternately transmits horizontal or vertical polarization microwaves, and then receives horizontal and vertical polarization backscattering waves simultaneously but separately with different modules. In this operation, however, the polarimetric characteristics of the processing signals are not always pure. In other words, the horizontal polarization signal is often mixed with some amount of vertical signal, or vice versa, both in transmitting and receiving, which is termed the crosstalk. The sources of crosstalk are mostly the TRMs and antenna systems [17].

The crosstalk error increases the magnitude of the cross-pol images relatively, and the target will be more likely to display volume scattering properties. Most PolSAR applications require that the crosstalk should be less than $-32 \mathrm{~dB}$ [18]. Meanwhile, for geophysical parameter inversion research and multitemporal studies over large areas, a higher accuracy is needed, and the crosstalk should be less than $-35 \mathrm{~dB}[19-21]$.

\subsection{Channel Imbalance Error}

Generally speaking, PolSAR technology discriminates ground objects by the coherence and difference of the four polarization channels. To guarantee that the coherence or difference only derives from the object's signature, the PolSAR systems should transmit or receive the polarimetric signals with the same antenna gain. The channel imbalance results from the antenna gain error, and it describes the amplitude or phase unconformity of the different polarization channels, both in transmitting and receiving [22].

According to the influence on the images, the channel imbalance can be divided into two parts, i.e., the co-polarization channel imbalance (co-imba) and the cross-polarization channel imbalance (cross-imba). The co-imba mainly causes the distortion between the co-pol channels, i.e., the $\mathrm{HH}$ (transmitting in horizontal polarization and receiving in horizontal polarization) and VV (transmitting in vertical polarization and receiving in vertical polarization) polarizations. The cross-imba mainly induces distortion between HV (transmitting in vertical polarization and receiving in horizontal polarization) and $\mathrm{VH}$ (transmitting in horizontal polarization and receiving in vertical polarization) polarizations, deforming the scattering reciprocity.

\subsection{Faraday Rotation Error}

When an electromagnetic wave propagates through an external magnetic field, the polarization plane rotates around the radar line of sight with the Faraday rotation angle (FRA). Considering the ionosphere layer and the geomagnetic field, the FRA is always considered for spaceborne SAR systems. 
The FRA is determined mostly by the transmission frequency, and it is also proportional to the total electron content (TEC) resulting from solar activity, the angle between the line of sight and geomagnetic, and also the geomagnetic flux density. The theoretical value is given by:

$$
\Omega=\frac{K}{f^{2}}\left\langle B \cdot \cos \varphi \cdot \sec \theta_{0} \cdot T E C\right\rangle
$$

where $K=2.365 \times 10^{4}$ in SI units, and is the transmission frequency (in Hz). TEC stands for the electrons per square meter, and parameter B is the geomagnetic flux density (in tesla). Term $\varphi$ is the angle between the geomagnetic field vector and the radar line of sight (in radians), and $\theta_{0}$ is the incidence angle. The symbol $\langle\cdot\rangle$ indicates an averaging operation.

The FRA can be estimated from the PolSAR data by the scattering reciprocity [23], but other polarimetric distortions may reduce the confidence. Thus, methods with external calibrators can calculate this parameter more accurately [24]. According to the current research, the FRA is apparent in low-frequency PolSAR systems such as the L- and P-band systems, but is not an issue for the C- or X-band satellites [9]. As GF-3 operates in the C-band, we do not discuss the FRA any further.

\subsection{Random System Noise Error}

System noise can arise from any part of the SAR system. It can result from electronic leakage or the physical temperature of the equipment. In most observation cases, the system noise is regarded as thermal noise, and obeys a Gaussian distribution of white noise characteristics. Therefore, the system noise is additive in PolSAR images, and is independent of the signal polarization. Using the scattering reciprocity hypothesis, the noise can be represented in the form of the signal-to-noise ratio (SNR):

$$
S N R=10 \cdot \log _{10}\left(\frac{\left|C_{22}\right|-\left|C_{23}\right|}{1 / 4 \cdot\left(C_{11}+C_{22}+C_{33}+C_{44}\right)}\right),
$$

where $C_{*}$ is the covariance matrix element transformed from the scattering matrix [25]. As the system noise can disturb the estimation accuracy of the other polarimetric distortions, it should be considered in PolCAL, although it cannot be eliminated, due to its random property.

\subsection{QualifyValue Adjustment}

The standard GF-3 multi-polarization images must be carried on QualifyValue adjustment before use. During the imaging processing, the numerical range of the observed data is rectified. However, the different channel images are rectified with different parameters, which will change the polarimetric properties. Furthermore, every image has different rectifying parameters. Therefore, before we proceed with any treatment of the GF-3 images, we need to check the QualifyValue parameters for each channel in the metadata, and undertake QualifyValue adjustment using the following formula:

$$
D N_{\text {true }}=D N_{\text {image }} * \text { QualifyValue/32767, }
$$

where the $D N_{\text {image }}$ term stands for the data in one polarization channel of GF-3 standard images. Parameter $D N_{\text {true }}$ is the true data we want to process for further research. QualifyValue is the scale parameter saved in the metadata, which is different for every polarization channel.

\section{Calibration Method and Processing}

Reflection symmetry and scattering reciprocity targets are distributed widely on the Earth's surface, and they also have some particular characteristics in mathematical expression. Accordingly, they are commonly explored in PolCAL, and are still utilized in calibration of the current PolSAR systems and PolCAL algorithm research. Considering this, we performed the PolCAL and accuracy 
assessment based on reflection symmetry and scattering reciprocity. Meanwhile, to assess the images more objectively, ground CRs were also used.

\subsection{PolCAL Method Based on Reflection Symmetry}

To suppress the randomness of the PolCAL errors and obtain stable signatures for the PolCAL reference targets, the calculation of crosstalk and cross-imba is carried out on the average covariance matrix of the image. It should also be noted that the distortion errors generally vary with the image range. Considering these factors, we divide the measured image into different strips along the image range, and generate the average covariance matrix. On the other hand, since the reflection symmetry property can provide stable constraints for PolCAL, it is reasonable to extract reference samples that satisfy reflection symmetry before calibration. There are some studies that focus on PolCAL samples extraction, and here we extract these samples by using helix scattering. The pixels with low helix scattering component will be extracted as PolCAL samples $[10,26]$. From the PolSAR distortion model in Equation (1), we obtain the vector form, and then gain the average covariance matrix [27]:

$$
\left[\begin{array}{c}
O_{h h} \\
O_{h v} \\
O_{v h} \\
O_{v v}
\end{array}\right]=A\left[\begin{array}{cccc}
1 & v & w & w v \\
z & 1 & w z & w \\
u & u v & 1 & v \\
u z & u & z & 1
\end{array}\right]\left[\begin{array}{cccc}
\alpha & 0 & 0 & 0 \\
0 & \alpha^{-1} & 0 & 0 \\
0 & 0 & \alpha & 0 \\
0 & 0 & 0 & \alpha^{-1}
\end{array}\right]\left[\begin{array}{cccc}
k & 0 & 0 & 0 \\
0 & 1 & 0 & 0 \\
0 & 0 & 1 & 0 \\
0 & 0 & 0 & k^{-1}
\end{array}\right]\left[\begin{array}{c}
S_{h h} \\
S_{h v} \\
S_{v h} \\
S_{v v}
\end{array}\right]+\left[\begin{array}{c}
N_{h h} \\
N_{h v} \\
N_{v h} \\
N_{v v}
\end{array}\right]
$$

$\Rightarrow[O]=A[P][F][K][S]+[N]$

$$
[M]=\left\langle\left[\begin{array}{c}
O_{h h} \\
O_{h v} \\
O_{v h} \\
O_{v v}
\end{array}\right] \cdot\left[\begin{array}{llll}
O_{h h}^{*} & O_{h v}^{*} & O_{v h}^{*} & O_{v v}^{*}
\end{array}\right]\right\rangle
$$

where matrix $P$ is the crosstalk distortion matrix, and matrix $F$ is the cross-imba matrix. Matrix $K$ represents the co-imba. These three matrices are also referred to as PDMs. The superscript ${ }^{*}$ means the complex conjugate operation, and matrix $M$ is a $4 \times 4$ complex matrix that represents the measured image.

The scattering mechanism of reflection symmetry mainly displayed by distributed targets such as grassland, desert, public squares, and bare soil. This means that the objects that satisfy this mechanism are symmetric by the line of sight in radiometric scattering. Theoretically, the covariance matrix of these targets is [25]:

$$
[C]=\left(\begin{array}{ccc}
\left\langle S_{h h} S_{h h}^{*}\right\rangle & 0 & \left\langle S_{h h} S_{v v}^{*}\right\rangle \\
0 & \left\langle S_{h v} S_{h v}^{*}\right\rangle & 0 \\
\left\langle S_{v v} S_{h h}^{*}\right\rangle & 0 & \left\langle S_{v v} S_{v v}^{*}\right\rangle
\end{array}\right)
$$

where the symbol $\langle\cdot\rangle$ indicates an averaging operation. The formula shows that the co-pol and cross-pol have zero coherence. Using the zero elements in the covariance matrix, and substituting them into the PolCAL distortion model, the PDMs can be calculated with the following formulas [27]:

$$
\begin{aligned}
u & =\left(M_{44} M_{21}-M_{41} M_{24}\right) /\left(M_{44} M_{11}-M_{41} M_{14}\right) \\
v & =\left(M_{11} M_{24}-M_{21} M_{14}\right) /\left(M_{44} M_{11}-M_{41} M_{14}\right) \\
z & =\left(M_{44} M_{31}-M_{41} M_{34}\right) /\left(M_{44} M_{11}-M_{41} M_{14}\right) \\
w & =\left(M_{11} M_{34}-M_{31} M_{14}\right) /\left(M_{44} M_{11}-M_{41} M_{14}\right),
\end{aligned}
$$

where $M_{*}$ is the element of the average covariance matrix M. Accordingly, the cross-imba is calculated with [5]:

$$
\alpha=\left(\frac{M_{32}-u^{*}\left(z M_{11}+w M_{41}\right)-v^{*}\left(z M_{14}+w M_{44}\right)}{M_{33}-z^{*}\left(z M_{11}+w M_{41}\right)-w^{*}\left(z M_{14}+w M_{44}\right)}\right)^{*} .
$$


Although there are some algorithms that calculate the co-imba by the use of natural targets such as bare soil [13], the prevailing and stable method relies on manmade calibrators. PARCs have a better signal-to-clutter ratio, but they are expensive. Triangular CRs are often used in PolCAL, and the parameter is calculated as:

$$
|k|=\left|\frac{O_{h h} O_{h h}^{*}}{O_{v v} O_{v v}^{*}}\right|^{\frac{1}{4}}, \arg (k)=\frac{1}{2} \arg \left(O_{h h} O_{v v}^{*}\right) .
$$

\subsection{PolCAL Method Based on Scattering Reciprocity}

Apart from some particular manmade targets, most scattering media conform to the reciprocity property, i.e., $S_{h v}=S_{v h}$, which leads to the following form of covariance matrix [16]:

$$
[C]=\left[\begin{array}{cccc}
C_{h h h h} & A^{*} & A^{*} & C_{h h v v} \\
A & \beta & \beta^{\prime} & B \\
A & \beta^{\prime} & \beta & B \\
C_{v v h h} & B^{*} & B^{*} & C_{v v v v}
\end{array}\right]
$$

in which the superscript * is the complex conjugate operator, the values of $\beta$ and $\beta^{\prime}$ are real, and the others are complex. $A$ and $B$ are the complex coherence between the co-pol and cross-pol channels. Since reciprocity implies that the two cross-pol channels are identical, for calibrated data, all of their correlations must in fact be identical.

The PolCAL algorithm based on scattering reciprocity is an iterative process, and is widely used in the present PolSAR systems. We only give the calculation of the polarimetric distortion parameters here, and more details can be found in [16].

This amplitude of cross-imba is calculated by the power of the two cross-polarizations, and the phase is calculated by their coherence:

$$
\alpha=\left(\left\langle M_{v h v h}\right\rangle /\left\langle M_{h v h v}\right\rangle\right)^{1 / 4} \exp \left(i * \operatorname{Angle}\left(\left\langle M_{v h h v}\right\rangle\right) / 2\right)
$$

In the equation, the symbol Angle $(\cdot)$ is the operator that calculates the phase of parameters. Subsequently, the calculated error is removed to generate the updated covariance matrix by matrix multiplication $\left[M^{\prime}\right]=[F]^{-1}[M][F]^{-H}$.

In every iteration, the crosstalk is calculated by the coherence of the cross-pol and co-pol channels, and the computation is based on the updated covariance matrix:

$$
\begin{aligned}
& \left(M_{v h h h}^{\prime}+M_{h v h h}^{\prime}\right) / 2 \cong\left(M_{v h h h}^{\prime}-\Delta u M_{h h h h}^{\prime}-\Delta v M_{v v h h}^{\prime}-\Delta v^{*} M_{v h h v}^{\prime}-\Delta w^{*} M_{v h v h}^{\prime}\right) \\
& \left(M_{v h h h}^{\prime}+M_{h v h h}^{\prime}\right) / 2 \cong\left(M_{h v h h}^{\prime}-\Delta w M_{v v h h}^{\prime}-\Delta z M_{h h h h}^{\prime}-\Delta v^{*} M_{h v h v}^{\prime}-\Delta w^{*} M_{h v v h}^{\prime}\right) \\
& \left(M_{v h v v}^{\prime}+M_{h v v v}^{\prime}\right) / 2 \cong\left(M_{v h v v}^{\prime}-\Delta u M_{h h v v}^{\prime}-\Delta v M_{v v v v}^{\prime}-\Delta u^{*} M_{v h h v}^{\prime}-\Delta z^{*} M_{v h v h}^{\prime}\right) \\
& \left(M_{v h v v}^{\prime}+M_{h v v v}^{\prime}\right) / 2 \cong\left(M_{h v v v}^{\prime}-\Delta w M_{v v v v}^{\prime}-\Delta z M_{h h v v}^{\prime}-\Delta u^{*} M_{h v h v}^{\prime}-\Delta z^{*} M_{h v v h}^{\prime}\right)
\end{aligned}
$$

In the formula, $\Delta u, \Delta v, \Delta w, \Delta z$ are the corrections of the crosstalk, and the update of the crosstalk can be depicted as:

$$
[u, v, w, z]^{T}=[u, v, w, z]^{T}+[\Delta u, \Delta v, \Delta w, \Delta z]^{T} .
$$

The calculations of the co-imba error and system noise are independent of the previous calculation, and their estimation is carried out in the same way as the reflection symmetry method. 


\section{Experiments}

\subsection{Experimental Data}

In this study, GF-3 satellite images of the full-polarimetric Strip I mode were used as the experimental data. The resolution of this imagery is $8 \mathrm{~m}$, and they are single look complex (SLC) images. To assess the image quality and verify the PolCAL improvement more objectively, 14 images from the two experimental sites were tested. The specific information is shown in Table 2.

Table 2. Specific parameters of the experimental GF-3 images.

\begin{tabular}{|c|c|c|c|c|}
\hline Imaging Region & Acquisition Date & Image ID & Central Look Angle & Image Size (Pixels) \\
\hline \multirow{10}{*}{ Etuoke Banner } & \multirow{2}{*}{12 June 2017} & 0002441640 & 39.98 & $5881 * 4717$ \\
\hline & & 0002441641 & 39.97 & $5778^{*} 4717$ \\
\hline & \multirow{2}{*}{24 June 2017} & 0002441191 & 43.92 & $7001 * 6161$ \\
\hline & & 0002441190 & 43.91 & $7054^{*} 6161$ \\
\hline & \multirow{2}{*}{06 July 2017} & 0002463755 & 47.45 & $3775 * 5977$ \\
\hline & & 0002463754 & 47.44 & $3814 * 5977$ \\
\hline & \multirow{2}{*}{11 July 2017} & 0002475549 & 40.01 & $6165^{*} 4708$ \\
\hline & & 0002475550 & 40.01 & $6206^{*} 4708$ \\
\hline & \multirow{2}{*}{16 July 2017} & 0002491702 & 30.43 & $6770 * 7182$ \\
\hline & & 0002491701 & 30.43 & $6778^{* 7182}$ \\
\hline \multirow{4}{*}{ Wuhan } & 30 April 2017 & 0002335427 & 36.21 & $7982 * 6200$ \\
\hline & 30 April 2017 & 0002335421 & 36.22 & $7944 * 6200$ \\
\hline & 24 August 2017 & 0002559955 & 36.15 & $7464 * 6209$ \\
\hline & 02 October 2017 & 0002645747 & 37.54 & $5821 * 5959$ \\
\hline
\end{tabular}

The first experimental area was Etuoke Banner of Inner Mongolia. Most of the land cover of this area is sparse grassland, which is perfect for PolCAL, and there are also some salt lakes, towns, farmland, and hillsides. In order to evaluate the stability of the satellite system, researchers from the Institute of Electronics, Chinese Academy of Sciences (IECAS), set up CRs and PARCs in this area in June and July 2017, obtaining valuable ground data. The city of Wuhan in Hubei province was also selected as an experimental area, and the land cover of this area mainly are buildings, water area, forests, and farmland.

\subsection{Experimental Design}

For the Etuoke Banner site, there were two images of the same orbit each day. One of the images had ground CRs, so we took this image to calculate the PDMs. We then calibrated both of the images with the same PDMs. We used the reflection symmetry method to PolCAL the images, and used both methods to assess the accuracy.

For the Wuhan site, there was only one image each day, and there was no manmade CR in the images. Generally, the methods described in this paper use distributed targets that satisfy certain assumptions to calculate crosstalk and cross-imba PDMs, and the methods use at least one manmade CR to estimate co-imba PDM. Therefore, we only evaluated the crosstalk and the cross-imba here. We calculated these two polarimetric errors in each image firstly to see the image quality, and then calibrated the images with the errors that were calculated by the same image.

\subsection{Experimental Results and Analysis}

Since the PDMs vary with range, we first divided the measured images into different strips along the image range. In this experiment, the size of the strips was $100^{*} \mathrm{Ncol}$ pixels, where the term ' $\mathrm{Ncol}^{\prime}$ means the size of the image in the azimuth direction. Secondly, we utilized an automatic extracting method to extract proper reference samples for PolCAL [10], and we then computed the average 
covariance matrices. After the PolCAL, we carried out the accuracy analysis on both the calibrated and uncalibrated images.

The polarization response signatures (PRS) of the CRs in the Etuoke Banner site are shown in Figure 1. There were more than five CRs in each image, and all of them were trihedral angle reflectors. We used three CRs to estimate the PDMs and one for verification. The PRS is widely used in PolCAL, and its main purpose is to check the correctness of the calibration. The PRS shows the normalized synthetic span value at different polarization patterns, which are expressed in a combination of ellipse orientation angel $\varphi$ (degree) and ellipticity angle $\chi$ (degree). The co-polarized response is synthesized when the polarization of emission wave and receiving wave are the same, and the cross-polarized response is synthesized when they are orthogonal [28].

The first PRS picture is the theoretical form of a triangular CR. There are two PRS pictures for each image: the left one is the PRSs before calibration, and the right one is the PRSs after calibration. The uncalibrated pictures show that there is still some polarimetric distortion in the images after the periodic systematic calibration. Secondly, some of the PRSs are much closer to the theoretical ones, which indicates that the periodic calibration is useful. On the other hand, the calibrated PRSs appear more closely to the theoretical form. After PolCAL, the peak lines of co-polarized PRSs fluctuate less, and the bottom lines of cross-polarized PRSs become straighter. This means that the further PolCAL processing can suppress the residual error and improve the polarimetric quality.
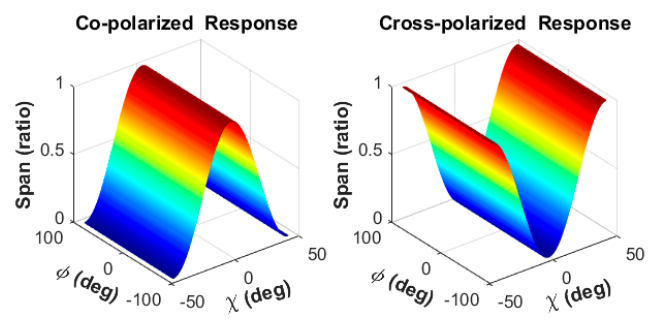

(a)

Co-polarized Response

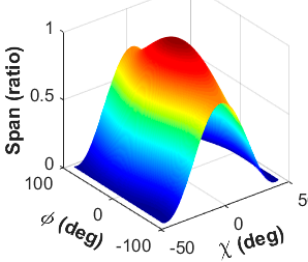

Co-polarized Response

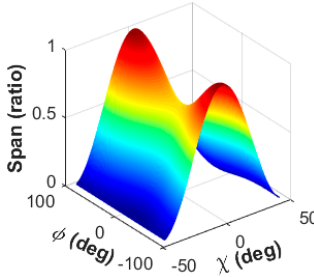

(d)
Cross-polarized Response

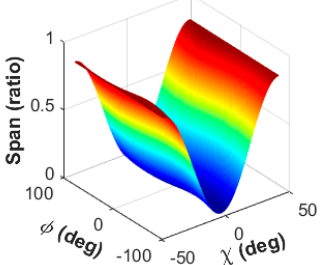

(b)

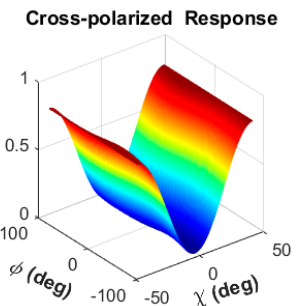

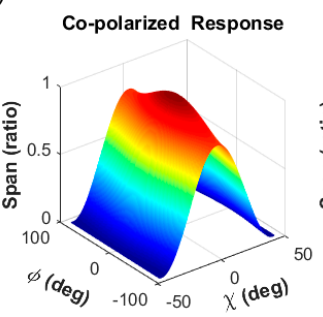

(c)

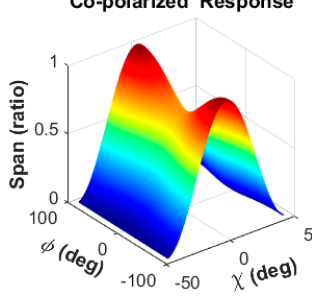

(e)
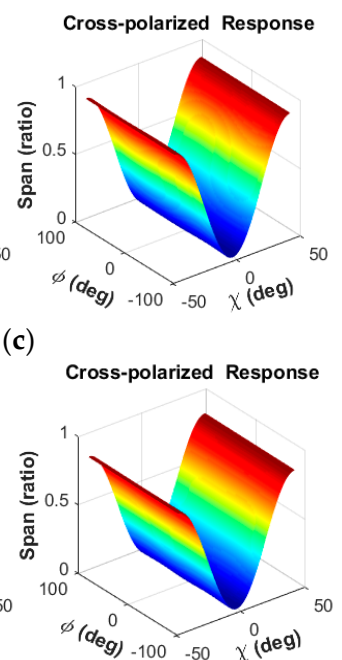

Figure 1. Cont. 


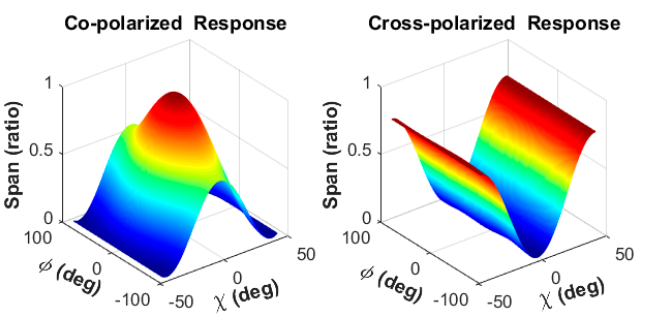

(f)

Co-polarized Response

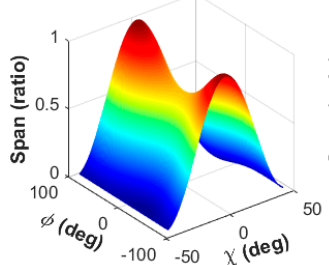

(h)

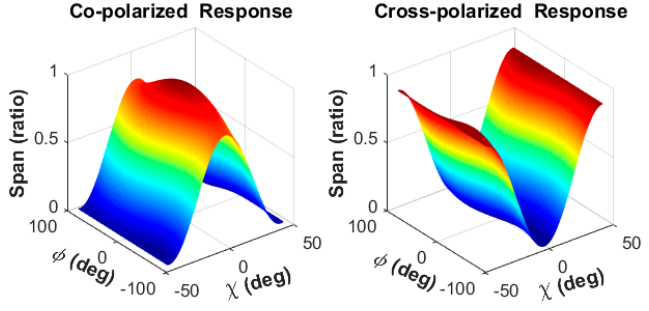

(j)

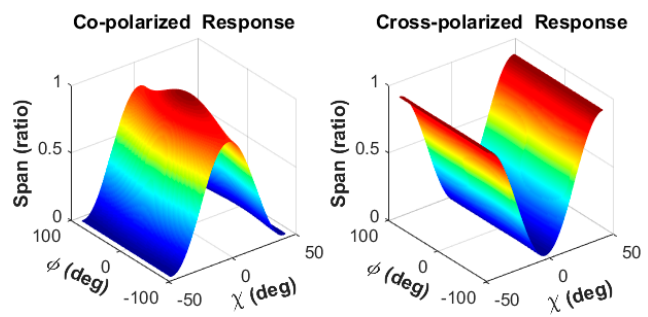

(g)

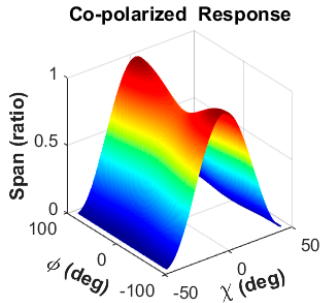

Cross-polarized Response

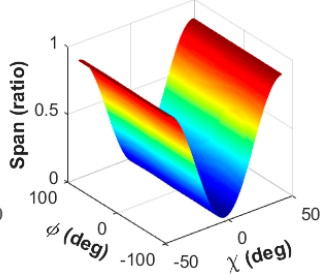

(i)
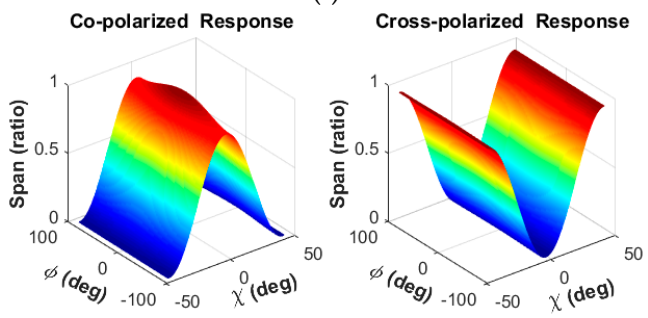

$(\mathbf{k})$

Figure 1. The co-pol and cross-pol response signature of CRs. The signatures show the normalized synthetic span value at different polarization pattern (expressed in a combination of ellipse orientation angel $\varphi$ and ellipticity angle $\chi$ ). These charts are: (a) theoretical PRS form of a triangular CR; $(\mathbf{b}, \mathbf{c})$ the PRSs before PolCAL and after PolCAL of image 0002441640; (d,e) the PRSs before PolCAL and after PolCAL of image 0002441191; (f,g) the PRSs before PolCAL and after PolCAL of image 0002463755; $(\mathbf{h}, \mathbf{i})$ the PRSs before PolCAL and after PolCAL of image 0002475549; $(\mathbf{j}, \mathbf{k})$ the PRSs before PolCAL and after PolCAL of image 0002491702. There are two PRS pictures for each image: the left one is the PRSs before calibration, and the right one is the PRSs after calibration.

The quantitative analysis and assessment were carried out on the PDMs. We estimated the residual PDMs and analyzed the polarimetric accuracy. Both the methods based on reflection symmetry and scattering reciprocity were performed. The polarization distortion errors of the GF-3 standard images are shown in Table 3. The crosstalk errors estimated by the two methods are different: the reflection symmetry method overestimates it (marked as Sym. in table), while the scattering reciprocity method underestimates it (marked as Rec. in table). Hence, we list both of the results separately. The channel imbalance errors calculated by these two methods are almost the same, so we show the average results. The co-imba errors were computed by CRs; therefore, there are only five images with these results. Generally speaking, the PDMs vary with the slant range, i.e., the incidence angle of the image, so we give here the mean value and standard deviation, for simplicity. The residual errors that exceed the accuracy requirement of the GF- 3 satellite (crosstalk $-35 \mathrm{~dB}$, channel imbalance $0.5 \mathrm{~dB}$, phase \pm 10 degrees) are marked in blue.

The results in Table 3 show that the channel isolation in GF-3 is controlled very well, and the crosstalk results of both methods satisfy the accuracy requirements. However, for the channel imbalance parameter, there are two values in one image exceeding the requirement slightly, and one value in one image is obviously abnormal. On the other hand, the system noise level of GF-3 is very low, in that the SNRs of all the images are better than $18 \mathrm{~dB}$. In this situation, the noise has no influence on the PDM estimation and will not decrease the image quality [29]. 
Table 3. Polarization distortion errors of the standard GF-3 images.

\begin{tabular}{llllcccc}
\hline Image ID & $\begin{array}{l}\text { Crosstalk- } \\
\text { Sym. (dB) }\end{array}$ & $\begin{array}{l}\text { Crosstalk- } \\
\text { Rec. (dB) }\end{array}$ & $|\boldsymbol{\alpha}| \mathbf{( d B})$ & $\begin{array}{c}\operatorname{Arg}(\boldsymbol{\alpha}) \\
(\mathbf{d e g})\end{array}$ & $\begin{array}{c}|\mathbf{k}| \\
(\mathbf{d B})\end{array}$ & $\begin{array}{c}\mathbf{A r g}(\mathbf{k}) \\
(\mathbf{d e g})\end{array}$ & $\begin{array}{c}\text { SNR } \\
(\mathbf{d B})\end{array}$ \\
\hline $\mathbf{0 0 0 2 4 4 1 6 4 0}$ & $-40 \pm 4.4$ & $-48 \pm 3.4$ & $-0.35 \pm 0.10$ & $3.6 \pm 0.3$ & 0.45 & -6.5 & 19 \\
$\mathbf{0 0 0 2 4 4 1 6 4 1}$ & $-45 \pm 3.4$ & $-51 \pm 1.9$ & $-0.20 \pm 0.05$ & $3.2 \pm 0.2$ & - & - & 19 \\
$\mathbf{0 0 0 2 4 4 1 1 9 1}$ & $-37 \pm 4.0$ & $-46 \pm 4.4$ & $-0.28 \pm 0.15$ & $0.8 \pm 0.3$ & -0.10 & -5.4 & 18 \\
$\mathbf{0 0 0 2 4 4 1 1 9 0}$ & $-36 \pm 2.9$ & $-46 \pm 2.5$ & $-0.22 \pm 0.04$ & $0.8 \pm 0.2$ & - & - & 19 \\
$\mathbf{0 0 0 2 4 6 3 7 5 5}$ & $-40 \pm 3.8$ & $-48 \pm 3.4$ & $0.55 \pm 0.19$ & $2.6 \pm 0.1$ & 0.57 & -2.3 & 20 \\
$\mathbf{0 0 0 2 4 6 3 7 5 4}$ & $-38 \pm 2.4$ & $-49 \pm 1.1$ & $0.48 \pm 0.01$ & $2.7 \pm 0.1$ & - & - & 19 \\
$\mathbf{0 0 0 2 4 7 5 5 4 9}$ & $-39 \pm 4.3$ & $-48 \pm 3.8$ & $-0.43 \pm 0.07$ & $-1.6 \pm 0.4$ & -0.16 & -7.4 & 18 \\
$\mathbf{0 0 0 2 4 7 5 5 5 0}$ & $-41 \pm 3.3$ & $-49 \pm 1.8$ & $-0.30 \pm 0.03$ & $-1.6 \pm 0.2$ & - & - & 19 \\
$\mathbf{0 0 0 2 4 9 1 7 0 2}$ & $-43 \pm 4.1$ & $-47 \pm 2.7$ & $-0.16 \pm 0.06$ & $3.5 \pm 0.4$ & 0.31 & -9.5 & 21 \\
$\mathbf{0 0 0 2 4 9 1 7 0 1}$ & $-46 \pm 3.9$ & $-47 \pm 1.3$ & $-0.18 \pm 0.03$ & $3.6 \pm 0.1$ & - & - & 20 \\
$\mathbf{0 0 0 2 3 3 5 4 2 7}$ & $-35 \pm 8.8$ & $-47 \pm 2.8$ & $-0.44 \pm 0.05$ & $-6.3 \pm 0.6$ & - & - & 18 \\
$\mathbf{0 0 0 2 3 3 5 4 2 1}$ & $-50 \pm 7.1$ & $-61 \pm 1.5$ & $1.04 \pm 0.02$ & $-6.5 \pm 0.5$ & - & - & 19 \\
$\mathbf{0 0 0 2 5 5 9 9 5 5}$ & $-37 \pm 7.6$ & $-51 \pm 4.2$ & $-0.39 \pm 0.04$ & $3.4 \pm 0.5$ & - & - & 19 \\
$\mathbf{0 0 0 2 6 4 5 7 4 7}$ & $-42 \pm 6.2$ & $-59 \pm 5.3$ & $-0.39 \pm 0.01$ & $7.6 \pm 0.1$ & - & - & 20 \\
\hline
\end{tabular}

We took the PolCAL processing of the standard GF-3 images and then re-estimated the residual errors to verify the effectiveness of the PolCAL method. We selected the reflection symmetry method for the PolCAL of the images, for its robustness. Furthermore, we estimated the residual errors using both of the methods. The results are shown in Table 4. We can see that, after PolCAL, the residual errors are substantially reduced for most of the cases. In addition, the crosstalk parameters that were estimated by the scattering reciprocity method at the Wuhan site are quite low. This because this method will underestimate the crosstalk, as described above, and the most objective way to evaluate image quality is using PARCs totally. On the other hand, these results are the best achievable residuals because they are obtained by calibrating each image with the PDMs computed from the image itself. However, two error values in image 0002441641 became larger than before (marked in red). This exception is a result of the experiment design. Since the polarimetric distortions are quite different between standard GF-3 image 0002441640 and image 0002441641, the deviation is generated when we calibrate image 0002441641 with the PDMs estimated by image 0002441640 . This situation will be avoided when we calibrate image with PDMs estimated by itself, as shown at the Wuhan site, i.e., the last four images. Apart from this, the crosstalk is below $-42 \mathrm{~dB}$ and the channel imbalance is better than $0.26 \mathrm{~dB}$ and \pm 0.2 degrees. This indicates that the PolCAL is effective, and it can clearly improve the image polarimetric quality.

Table 4. Polarization residual errors after PolCAL.

\begin{tabular}{cccccccc}
\hline Image ID & $\begin{array}{c}\text { Crosstalk- } \\
\text { Sym. (dB) }\end{array}$ & $\begin{array}{l}\text { Crosstalk- } \\
\text { Rec. (dB) }\end{array}$ & $|\boldsymbol{\alpha}| \mathbf{( d B})$ & $\begin{array}{c}\operatorname{Arg}(\boldsymbol{\alpha}) \\
(\mathbf{d e g} .)\end{array}$ & $\begin{array}{c}|\mathbf{k}| \\
(\mathbf{d B})\end{array}$ & $\begin{array}{c}\operatorname{Arg}(\mathbf{k}) \\
(\mathbf{d e g})\end{array}$ & $\begin{array}{c}\text { SNR } \\
(\mathbf{d B})\end{array}$ \\
\hline $\mathbf{0 0 0 2 4 4 1 6 4 0}$ & $-50 \pm 6.8$ & $-66 \pm 10.5$ & $0.07 \pm 0.07$ & $-0.1 \pm 0.3$ & -0.05 & -0.1 & 19 \\
$\mathbf{0 0 0 2 4 4 1 6 4 1}$ & $-40 \pm 3.1$ & $-54 \pm 3.1$ & $0.25 \pm 0.07$ & $-0.3 \pm 0.4$ & - & - & 19 \\
$\mathbf{0 0 0 2 4 4 1 1 9 1}$ & $-50 \pm 6.8$ & $-64 \pm 9.8$ & $0.10 \pm 0.10$ & $0.1 \pm 0.3$ & -0.05 & 0.01 & 18 \\
$\mathbf{0 0 0 2 4 4 1 1 9 0}$ & $-42 \pm 5.4$ & $-62 \pm 7.3$ & $0.09 \pm 0.08$ & $-0.03 \pm$ & - & - & 19 \\
& & & 0.2 & & & \\
$\mathbf{0 0 0 2 4 6 3 7 5 5}$ & $-52 \pm 6.2$ & $-69 \pm 9.5$ & $-0.10 \pm 0.14$ & $-0.01 \pm$ & 0.06 & -0.01 & 20 \\
$\mathbf{0 0 0 2 4 6 3 7 5 4}$ & $-45 \pm 5.0$ & $-66 \pm 7.6$ & $-0.14 \pm 0.03$ & $0.1 \pm 0.1$ & - & - & 19 \\
$\mathbf{0 0 0 2 4 7 5 5 4 9}$ & $-47 \pm 7.8$ & $-65 \pm 12.5$ & $0.10 \pm 0.04$ & $0.04 \pm 0.3$ & -0.06 & 0.01 & 18 \\
$\mathbf{0 0 0 2 4 7 5 5 5 0}$ & $-46 \pm 4.6$ & $-60 \pm 7.2$ & $0.26 \pm 0.06$ & $0.1 \pm 0.3$ & - & - & 19 \\
$\mathbf{0 0 0 2 4 9 1 7 0 2}$ & $-58 \pm 6.8$ & $-69 \pm 10.3$ & $0.04 \pm 0.05$ & $0.04 \pm 0.4$ & -0.02 & 0.01 & 21 \\
$\mathbf{0 0 0 2 4 9 1 7 0 1}$ & $-51 \pm 6.7$ & $-66 \pm 5.9$ & $0.02 \pm 0.04$ & $0.2 \pm 0.1$ & - & - & 20 \\
$\mathbf{0 0 0 2 3 3 5 4 2 7}$ & $-45 \pm 7.2$ & $-78 \pm 4.6$ & $0.07 \pm 0.01$ & $0.01 \pm 0.01$ & - & - & 18 \\
$\mathbf{0 0 0 2 3 3 5 4 2 1}$ & $-67 \pm 6.4$ & $-98 \pm 5.7$ & $-0.14 \pm 0.01$ & $-0.01 \pm$ & - & - & 19 \\
$\mathbf{0 0 0 2 5 5 9 9 5 5}$ & $-46 \pm 8.4$ & $-81 \pm 4.7$ & $0.07 \pm 0.01$ & $0.01 \pm 0.01$ & - & - & 19 \\
$\mathbf{0 0 0 2 6 4 5 7 4 7}$ & $-48 \pm 6.4$ & $-90 \pm 5.8$ & $0.05 \pm 0.01$ & $0.01 \pm 0.01$ & - & - & 20 \\
\hline
\end{tabular}




\section{Conclusions}

The GF-3 satellite is the first multi-polarization SAR imaging satellite in China for civil use, and it has three full-polarization imaging modes, for which the image polarimetric quality is vital for the increasing PolSAR research group and applications. In this study, we assessed the image polarimetric quality and carried out a PolCAL experiment to improve the accuracy. PolCAL methods based on both reflection symmetry and scattering reciprocity were analyzed and utilized. The experimental results show that the polarimetric accuracy of these GF-3 images is high, and it can meet the accuracy requirements practically. The experiments also indicate that the PolCAL processing of the standard GF-3 images can clearly improve the image polarimetric quality, with crosstalk of $-42 \mathrm{~dB}$ and channel imbalance of $0.26 \mathrm{~dB}$ in amplitude and \pm 0.2 degrees in phase.

Acknowledgments: The research is founded by the National Natural Science Foundation of China, Grant No. 41771377, No. 41501382, and No. 41601355; and the National Key Research and Development Program of China, under Grant 2016YFB0502603.

Author Contributions: Yonglei Chang carried out the experiment and wrote the paper. Pingxiang Li guided the experiment. Jie Yang guided the structure of the paper. Jinqi Zhao and Lingli Zhao checked the paper and provided suggestions. Ground control data was provided by Lei Shi.

Conflicts of Interest: The authors declare no conflict of interest.

\section{References}

1. Zhang, Q. System design and key technologies of the GF-3 satellite. Acta Geod. Cartogr. Sin. 2017, 46, 269-277.

2. Qingjun, Z.; Yadong, L. Overview of chinese first c band multi-polarization SAR satellite GF-3. Aerosp. China 2017, 3, 22-31.

3. Wang, C.; Liao, G.; Zhang, Q. First spaceborne SAR-GMTI experimental results for the chinese gaofen-3 dual-channel SAR sensor. Sensors 2017, 17, 2683. [CrossRef] [PubMed]

4. Zhao, J.; Yang, J.; Lu, Z.; Li, P.; Liu, W.; Yang, L. A novel method of change detection in bi-temporal polSAR data using a joint-classification classifier based on a similarity measure. Remote Sens. 2017, 9, 846. [CrossRef]

5. Van Zyl, J.J. Synthetic Aperture radar Polarimetry; John Wiley \& Sons: New York, NY, USA, 2011; Volume 2.

6. Yin, J.; Yang, J.; Zhang, Q. Assessment of GF-3 polarimetric SAR data for physical scattering mechanism analysis and terrain classification. Sensors 2017, 17, 2785. [CrossRef] [PubMed]

7. Canadian Space Agency. Available online: http://www.asc-csa.gc.ca/eng/satellites/radarsat/radarsattableau.asp (accessed on 27 January 2018).

8. Calibration Result of ALOS-2. Available online: http://www.eorc.jaxa.jp/ALOS-2/en/calval/calval_index.htm (accessed on 27 January 2018).

9. Shimada, M. Model-based polarimetric SAR calibration method using forest and surface-scattering targets. IEEE Trans. Geosci. Remote Sens. 2011, 49, 1712-1733. [CrossRef]

10. Chang, Y.; Yang, J.; Li, P.; Zhao, L.; Shi, L. Sample extraction based on helix scattering for polarimetric SAR calibration. ISPRS Inter. Arch. Photogramm. Remote Sens. 2017, XLII-2/W7, 689-692. [CrossRef]

11. Whitt, M.W.; Ulaby, F.T.; Polatin, P.; Liepa, V.V. A general polarimetric radar calibration technique. IEEE Trans. Antennas Propag. 1991, 39, 62-67. [CrossRef]

12. Sarabandi, K. Calibration of a polarimetric synthetic aperture radar using a known distributed target. IEEE Trans. Geosci. Remote Sens. 1994, 32, 575-582. [CrossRef]

13. Shi, L.; Yang, J.; Li, P. Co-polarization channel imbalance determination by the use of bare soil. ISPRS J. Photogramm. Remote Sens. 2014, 95, 53-67. [CrossRef]

14. Villa, A.; Iannini, L.; Giudici, D.; Monti-Guarnieri, A.; Tebaldini, S. Calibration of SAR polarimetric images by means of a covariance matching approach. IEEE Trans. Geosci. Remote Sens. 2015, 53, 674-686. [CrossRef]

15. Luscomb, A.P. Polarimetric parameter estimation from amazon images. In Proceedings of the CEOS WGCVSAR Workshop 2001, Tokyo, Japan, 2-5 April 2001. Available online: ftp:/ /ftp.eorc.jaxa.jp/cdroms/EORC061/data/f_papers/ceos078.pdf (accessed on 27 January 2018).

16. Ainsworth, T.L.; Ferro-Famil, L.; Lee, J.-S. Orientation angle preserving a posteriori polarimetric SAR calibration. IEEE Trans. Geosci. Remote Sens. 2006, 44, 994-1003. [CrossRef]

17. Woodhouse, I.H. Introduction to Microwave Remote Sensing; CRC press: Boca Rtaon, FL, USA, 2005. 
18. Xu, F.; Wang, H.; Jin, Y.-Q.; Liu, X.; Wang, R.; Deng, Y. Impact of cross-polarization isolation on polarimetric target decomposition and target detection. Radio Sci. 2015, 50, 327-338. [CrossRef]

19. Recommendations from CEOS CAL/VAL 2004. Available online: http:/ / earth.esa.int/workshops/ceos_ sar_2004/recommendation_at_ceos_cal.html (accessed on 27 January 2018).

20. Wang, C.; Yu, W.; Wang, Y.; Yan, H. Polarimetric calibration requirements on several classification schemes for land application of polarimetric synthetic aperture radar. IET Radar Sonar Navig. 2013, 7, 113-122. [CrossRef]

21. Kimura, H. Calibration of polarimetric palsar imagery affected by faraday rotation using polarization orientation. IEEE Trans. Geosci. Remote Sens. 2009, 47, 3943-3950. [CrossRef]

22. Fore, A.G.; Chapman, B.D.; Hawkins, B.P.; Hensley, S.; Jones, C.E.; Michel, T.R.; Muellerschoen, R.J. Uavsar polarimetric calibration. IEEE Trans. Geosci. Remote Sens. 2015, 53, 3481-3491. [CrossRef]

23. Bickel, S.H.; Bates, R.H.T. Effects of magneto-ionic propagation on the polarization scattering matrix. Proc. IEEE 1965, 53, 1089-1091. [CrossRef]

24. Quegan, S.; Lomas, M.R. The interaction between faraday rotation and system effects in synthetic aperture radar measurements of backscatter and biomass. IEEE Trans. Geosci. Remote Sens. 2015, 53, 4299-4312. [CrossRef]

25. Lee, J.-S.; Pottier, E. Polarimetric Radar Imaging: From Basics to Applications; CRC Press: Boca Raton, FL, USA, 2009; pp. 66-72.

26. Kimura, H.; Mizuno, T.; Papathanassiou, K.P.; Hajnsek, I. Improvement of polarimetric SAR calibration based on the quegan algorithm. In Proceedings of the 2004 IEEE International Geoscience and Remote Sensing Symposium, Anchorage, AK, USA, 20-24 September 2004; Volume 1, pp. 184-187.

27. Quegan, S. A unified algorithm for phase and cross-talk calibration of polarimetric data-theory and observations. IEEE Trans. Geosci. Remote Sens. 1994, 32, 89-99. [CrossRef]

28. Agrawal, A.P.; Boerner, W.-M. Redevelopment of kennaugh's target characteristic polarization state theory using the polarization transformation ration formalism for the coherent case. IEEE Trans. Geosci. Remote Sens. 1989, 27, 2-14. [CrossRef]

29. Chen, J.; Guo, W.; Li, Z.; Liu, W. Optimised limit for polarimetric calibration of fully polarised sar systems. Electron. Lett. 2016, 52, 763-764. [CrossRef] 